\title{
Pull-in Instability of Micro-switch Actuators: Model Review
}

\author{
Wen-Hui Lin ${ }^{1}$ and Ya-Pu Zhao ${ }^{2, *}$ \\ 1 College of Science, China Agricultural University, Beijing 100083, China \\ 2 State Key Laboratory of Nonlinear Mechanics (LNM), Institute of Mechanics, Chinese \\ Academy of Sciences, Beijing 100080, China
}

\begin{abstract}
The existing three widely used pull-in theoretical models (i.e., one-dimensional lumped model, linear supposition model and planar model) are compared with the nonlinear beam mode in this paper by considering both cantilever and fixed-fixed type micro and nano-switches. It is found that the error of the pull-in parameters between one-dimensional lumped model and the nonlinear beam model is large because the denominator of the electrostatic force is minimal when the electrostatic force is computed at the maximum deflection along the beam. Since both the linear superposition model and the slender planar model consider the variation of electrostatic force with the beam's deflection, these two models not only are of the same type but also own little error of the pull-in parameters with the nonlinear beam model, the error brought by these two models attributes to that the boundary conditions are not completely satisfied when computing the numerical integration of the deflection.
\end{abstract}

Keywords: Pull-in, micro and nano-switches, error estimation, theoretical models

\section{Introduction}

The pull-in phenomenon is widely applied in many micro, nano and quantum-machined actuators that require bi-stability for their operations [1-24]. A typical micro-switch is constructed from two conducting electrodes, one is fixed and the other is movable. The upper movable electrode will deflect downward to the ground electrode due to the electrostatic attraction after the application of a voltage difference between the two electrodes. The movable electrode becomes unstable and pull-in onto the ground electrode at a certain voltage. The voltage and deflection of the switch at this state are called the pull-in parameters or critical pull-in values of the switch $[1,11]$.

There are lots of studies focusing on modeling of the electrical pull-in phenomenon [1-10]. The simplest and easiest models for common use are the so-called one-dimensional (1D) ones. These models provide direct, fast and relative accurate estimations in the case of actuators with a well-defined single degree of freedom (1DOF) [1-8]. The 1D model was first put forward by Osterberg [1,2], and he obtained the analytical expression of the pull-in parameters about MEMS switches. This model is also adapted to investigate the influence of different intermolecular forces, such as van der Waals force [14,15], Casimir force [18,21,22] and capillary force [19], on the pull-in behavior of NEMS structure, from the stationary behavior to the dynamical behavior [11-16]. The model is able to qualitatively explain the unstable scenery and quantify the pull-in voltage, at which the electrical force overwhelms the restoring force. However, the spatial variation of the restoring force is ignored in the analysis and the pull-in voltage is overestimated. By considering the

\footnotetext{
* Author to whom any correspondence should be addressed. E-mail: yzhao@imech.ac.cn (Ya-Pu Zhao).
} 
axial force [3], the pull-in voltage is analyzed using the effective stiffness in [17]. Since we are only interested in the maximum deflection, another $1 \mathrm{DOF}$ model is using the integral method. That is, the distributed load is treated as superposition of the infinite number of concentrated forces, then the maximum deflection is obtained by integrating the deflection equation [4]. Another model is a lumped two degrees of freedom (L2DOF) model [10], which introduced a linear displacement and an angular displacement. The L2DOF model benefits from higher accuracy than the 1DOF model. The MEMS/NEMS switches are simplified by all these models as a beam model. As a common knowledge, beam model is the special situation of the plane model, and then we can use the plane theory to discuss this problem $[5,6]$. Here, the load is taken as distributed forces which are different everywhere along the axis. The axial effect is considered in [7-9]. These models are summarized in [10] and an improved model was put forward by considering the residual stress and fringing field effect.

To the best of authors' knowledge, these theoretical models are used by many researchers without comparison. In this paper, these theoretical models will be compared with the nonlinear beam model omitting the residual stress and fringing field effect, and the cantilever and fixed-fixed structures are considered.

\section{Comparison of different models for the cantilever switch}

\subsection{D lumped model [1,2]}

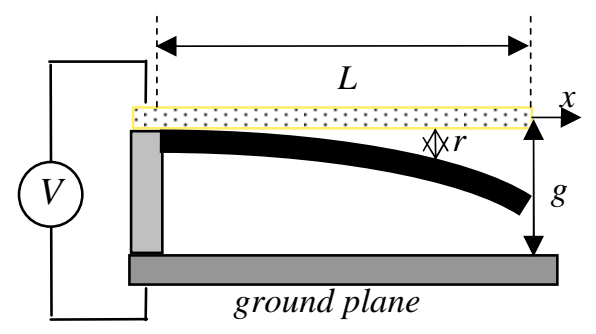

(a)
MEMS switches are generally modeled as a cantilever-type and fixed-fixed beam-type structures $[25,26]$. A micro-cantilever switch in Fig. 1(a) was simplified to a 1D lumped model as shown in Fig. 2 by Osterberg [1]. This model consists of a linear spring, a mass, and a parallel-beam capacitor. Only $1 \mathrm{DOF}$ of the system is the deflection of the free end, $r$, of the upper movable beam from its equilibrium position at $r=0$.

Neglecting the fringing fields, the electrostatic force for the parallel beam configuration shown in Fig. 2 is given as

$$
F_{\text {elec }}=\frac{\varepsilon_{0} w L V^{2}}{2(g-r)^{2}}
$$

where $\varepsilon_{0}$ is the permittivity of vacuum, $w$ and $L$ are the width and length of the beam, respectively, $g$ is the initial gap distance between the beam and the ground plane, and $V$ is the applied voltage. The restoring force of the beam is assumed to take the standard mass-spring form

$$
F_{\text {res }}=k(g-r)
$$

where $k$ is the spring constant for the beam and equals to $8 E I / L^{3}$ for a cantilever structure, $E$ is the Young's modulus, $I=w h^{3} / 12$ is the moment of inertia, and $h$ is the thickness of the beam.

The electrostatic force is attractive, while the restoring force is repulsive. Then we obtain the stationary equilibrium equation of this model as

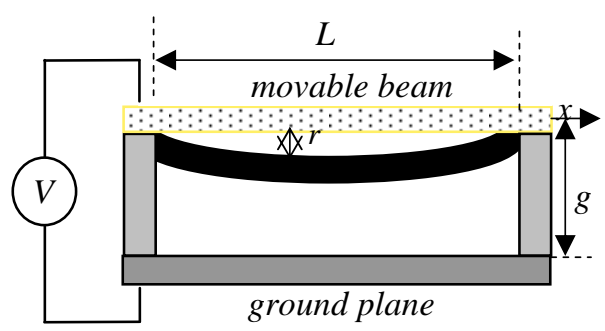

(b)

Figure 1. Schematic of MEMS switches: (a) cantilever-type, and (b) fixed-fixed beam-type. 


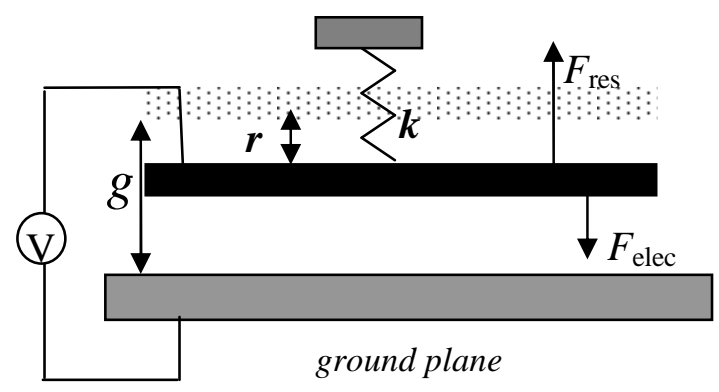

Figure 2. 1D lumped model for estimating pull-in parameters.

$$
F_{\text {res }}-F_{\text {elec }}=k r-\frac{\varepsilon_{0} w L V^{2}}{2(g-r)^{2}}=0 .
$$

Introducing dimensionless variable, $\Delta=r / g$, we get a corresponding dimensionless variable $a=\varepsilon_{0} w L V^{2} /\left(2 k g^{3}\right)$, which denotes the order of magnitude of ratio between the electrostatic and the restoring forces. Substituting $k=8 E I / L^{3}$ into the expression of $a$, we get $a=\varepsilon_{0} w V^{2} L^{4} /\left(16 E \operatorname{Ig}^{3}\right)$. As in Refs. [13,15], we can discuss variation of parameter $a$ instead of that of voltage $V$ for the given dimensions of this model, and we get the dimensionless equation as

$$
f(\Delta, a)=\Delta-\frac{a}{(1-\Delta)^{2}}=0 .
$$

According to the definition of these parameters, physically meaningful solutions exist in the region $0<\Delta<1$. The variation of the parameter $a$ with the maximum deflection $\Delta$ is shown in Fig. 4. By the critical condition $\partial f(\Delta) / \partial \Delta=0$ [27], one has

$$
\Delta_{\mathrm{PI}}=\frac{1}{3} \text {, and } a_{\mathrm{PI}}=\frac{4}{27},
$$

which are the same as the expressions derived in Refs. [1, 2].

\subsection{Linear superposition model [4]}

In this model, the effect of the different distributed load along the axis of the beam to the maximum deflection is considered. By using the linear superposition equation, the contributed load is treated as the superposition of the infinite number of concentrated forces. Since we only interested in the maximum deflection, we can use the following equation

$$
r(L)=\int_{0}^{L} \frac{x^{2}(3 L-x) q_{\mathrm{elec}}(x)}{6 E I} \mathrm{~d} x .
$$

In the above equation, the only new variable is $q_{\text {elec }}(x)$, the force per unit area, and $x$, the variable of integration along axial direction. In this paper, the electrostatic force (neglecting fringing fields) per unit area is

$$
q_{\mathrm{elec}}(x)=\frac{\varepsilon_{0} w V^{2}}{2(g-r(x))^{2}} .
$$

Combining Eqs. (6) and (7), we get

$$
r(L)=\int_{0}^{L} \frac{\varepsilon_{0} w V^{2}}{2(g-r(x))^{2}} \frac{x^{2}(3 L-x)}{6 E I} \mathrm{~d} x .
$$

Introduce the dimensionless variables as in Section 2.1, $s=x / L, u(s)=r(x) / g$, and $\Delta=u(1)=r(L) / g$, then we get

$$
\Delta=a \int_{0}^{1} \frac{4 s^{2}(3-s)}{3(1-u(s))^{2}} \mathrm{~d} s .
$$

The suggested form for $u(s)$ is a square-law curvature for the beam [4]

$$
u(s) \approx s^{2} \Delta \text {. }
$$

A part of boundary conditions are just satisfied by this assumption for $u(s)$. A solvable formula of $\Delta$ can be derived by combining Eqs. 
(9) and (10), that is,

$$
\Delta=a \int_{0}^{1} \frac{4 s^{2}(3-s)}{3\left(1-s^{2} \Delta\right)^{2}} \mathrm{~d} s .
$$

The above equation can be integrated as

$$
\begin{aligned}
\Delta= & a\left[\frac{1}{3 \Delta \sqrt{\Delta}}\left(3-\frac{2}{\sqrt{\Delta}}\right) \ln (1-\sqrt{\Delta})-\right. \\
& \left.\frac{1}{3 \Delta \sqrt{\Delta}}\left(3+\frac{2}{\sqrt{\Delta}}\right) \ln (1+\sqrt{\Delta})+\frac{4}{3 \Delta(1-\Delta)}\right]
\end{aligned} .
$$

The dimensionless variable $a$ can be calculated numerically for all values of $\Delta$, which has a fixed range between 0 and 1 . This relationship is shown in Fig. 3.

For Eq. (11), a numerical integration (Matlab) can be performed for every value of $\Delta$ between 0 and 1, the results are shown in Figs. 3 and 4. From Fig. 3, it is seen that the numerical solution is well consistent with the accurate solution. Then, the numerical solution can be obtained in the following discussion, and it is not need to integrate the corresponding equation.

According to the numerical computation, the critical values are obtained as follows

$$
\Delta_{\mathrm{PI}}=0.47 \text { and } a_{\mathrm{PI}}=0.2284 \text {. }
$$

\subsection{Planar theory [5]}

For narrow beams $(w<5 h)$, the equilibrium equation is

$$
E I \frac{\mathrm{d}^{4} r}{\mathrm{~d} x^{4}}=q_{\mathrm{elec}}(x)
$$

For convenience, the model is parameterized in the non-dimensional form as before, we have

$$
\frac{\mathrm{d}^{4} u}{\mathrm{~d} s^{4}}=a \frac{8}{(1-u)^{2}}=q(s) .
$$

The associated boundary conditions are

$$
u(0)=\frac{\mathrm{d} u}{\mathrm{~d} s}(0)=0, \frac{\mathrm{d}^{2} u}{\mathrm{~d} s^{2}}(1)=\frac{\mathrm{d}^{3} u}{\mathrm{~d} s^{3}}(1)=0
$$

This boundary-value problem can be solved easily by the variational iteration method [28-31].

Green's function is introduced by [5] to investigate the pull-in parameters. Then the deflection of the cantilever tip is

$$
\Delta=\frac{4 a}{3} \int_{0}^{1} \frac{s^{2}(3-s)}{(1-u(s))^{2}} \mathrm{~d} s
$$

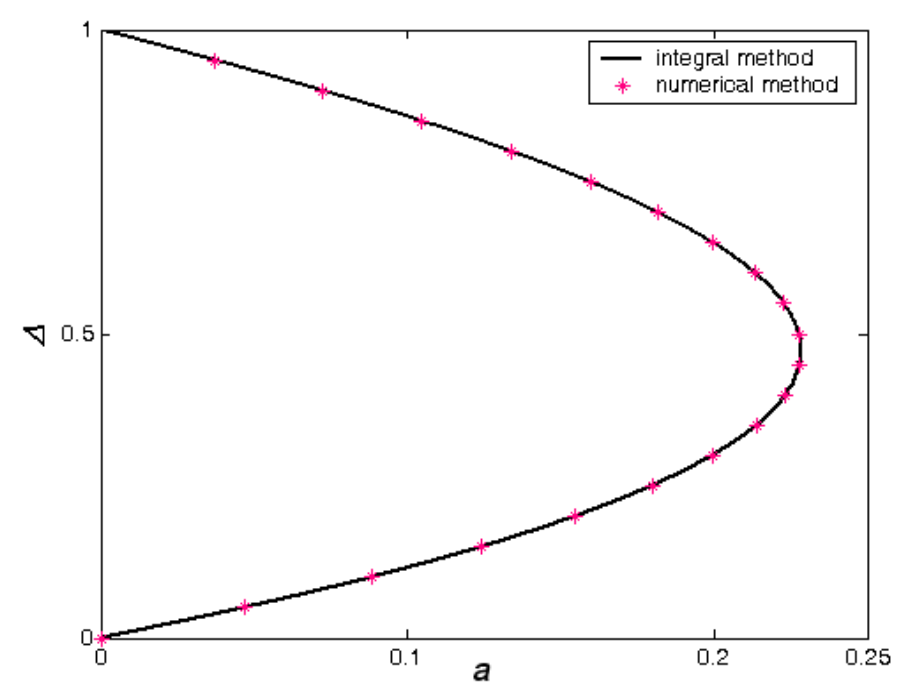

Figure 3. Comparison between the integral and the numerical methods for the linear superposition model. 


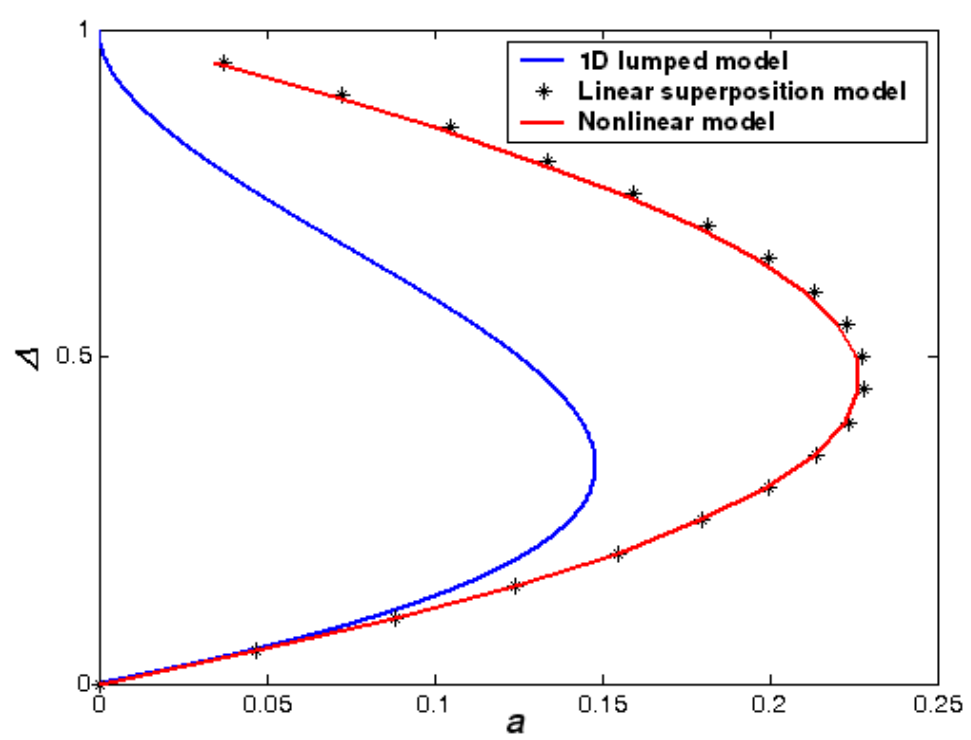

Figure 4. Variation of the maximum deflection $\Delta$ with the parameter $a$ of three different models for the cantilever switch.

This result is the same as Eq. (9). From these results, it is known that these two models can get the same results. As a result, the linear superposition model is only used in the following discussion.

\subsection{The nonlinear model of beam theory}

Linearized equations are adopted by the above three models. In this section, the nonlinear Euler-Bernoulli beam equation will be used directly

$$
E I \frac{r^{\prime \prime}(x)}{\left[1+r^{\prime}(x)^{2}\right]^{3 / 2}}=M(x),
$$

to get the pull-in parameters.

Substituting the corresponding moment and the dimensionless variables as in Section 2.2, then we get

$$
\frac{u^{\prime \prime}(s)}{\left[1+u^{\prime}(s)^{2}\right]^{3 / 2}}=a \int_{s}^{1} \frac{8(t-s)}{(1-u(t))^{2}} \mathrm{~d} t .
$$

To make $u(s)$ satisfy all of the boundary conditions, we assume the form of $u(s)$ as

$$
u(s)=a s^{4}+b s^{3}+c s^{2}+d s+e,
$$

the corresponding boundary conditions are:

$$
u(0)=u^{\prime}(0)=0, u^{\prime \prime}(1)=u^{\prime \prime \prime}(1)=0 .
$$

To determine the five undetermined constants $a, b, c, d, e$, we should add another condition,

$$
u(1)=\Delta .
$$

Substituting Eqs. (21) and (22) into Eq. (20), we obtain

$$
u(s)=\frac{\Delta}{3} s^{2}\left(s^{2}-4 s+6\right) .
$$

Set $s=0$ into Eq. (19) to simplify the computation, that is

$$
\frac{u^{\prime \prime}(0)}{\left[1+u^{\prime}(0)^{2}\right]^{3 / 2}}=a \int_{0}^{1} \frac{8 t}{(1-u(t))^{2}} \mathrm{~d} t
$$

From Eq. (23), we know that $u^{\prime \prime}(0)=4 \Delta$ and $u^{\prime}(0)=0$. Substituting these two values and Eq. (23) into Eq. (24), we have 


$$
4 \Delta=a \int_{0}^{1} \frac{8 s}{\left(1-\frac{\Delta}{3} s^{2}\left(s^{2}-4 s+6\right)\right)^{2}} \mathrm{ds}
$$

Using Matlab program, we can obtain the variation of the parameter $a$ with respect to the maximum deflection $\Delta$ between 0 and 1 , the result is shown in Fig. 4. At the same time, we obtain the corresponding critical values as follows

$$
\Delta_{\mathrm{PI}}=0.47 \text { and } a_{\mathrm{PI}}=0.2267 \text {. }
$$

\section{Comparison of different models for the fixed-fixed switch}

\subsection{D Linear model}

As in Section 2.1, the dimensionless equation of this model is still as follows

$$
g(\Delta, a)=\Delta-\frac{a}{(1-\Delta)^{2}}=0,
$$

where $a=\varepsilon_{0} w V^{2} L^{4} /\left(768 E I g^{3}\right)$ for the fixed-fixed structure. The variation of the parameter $a$ with the maximum deflection $\Delta$ is shown in Fig. 5. Similarly, using the critical condition [27], we get the same result as (5).

\subsection{Linear superposition model}

According to the basic equation in Elasticity, the expression of the maximum deflection for the fixed-fixed structure is obtained by directly substituting $q_{\text {elec }}(x)$, and introducing the dimensionless variables, the final result is

$$
\Delta=a \int_{0}^{1} \frac{2 s^{2}(3-2 s)}{(1-u(s))^{2}} \mathrm{~d} s=a \int_{0}^{1} \frac{2 s^{2}(3-2 s)}{(1-s(2-s) \Delta)^{2}} \mathrm{~d} s .
$$

Here, we should notice $s=2 x / L$, $\Delta=u(1)=r(L / 2) / g$, and $u(s) \approx s(2-s) \Delta$.
For Eq. (28), a numerical integration (Matlab) is used to compute the variation of the parameter $a$ with the maximum deflection $\Delta$ in Fig. 5, and the two critical values are obtained as

$$
\Delta_{\mathrm{PI}}=0.38 \text { and } a_{\mathrm{PI}}=0.1675 \text {. }
$$

\subsection{The nonlinear model of beam theory}

For the fixed-fixed beam, similarly in Section 2.3 , we get

$$
\begin{aligned}
\frac{u^{\prime \prime}(s)}{\left[1+\frac{2 g}{L} u^{\prime}(s)^{2}\right]^{3 / 2}}= & 6 a\left[\int_{0}^{2} \frac{\left(2 s-t^{2}-2\right) \mathrm{d} t}{(1-u(t))^{2}}\right. \\
& \left.-\int_{s}^{2} \frac{4(t-s) \mathrm{d} t}{(1-u(t))^{2}}\right]
\end{aligned}
$$

The variables are the same as in Section 3.2. Substituting the boundary conditions

$$
u(0)=u^{\prime}(0)=0, u(2)=u^{\prime}(2)=0,
$$

and

$$
u(1)=\Delta,
$$

into Eq. (20), we get

$$
u(s)=\Delta s^{2}(s-2)^{2} .
$$

Substituting Eq. (33) into Eq. (30) and setting $s=1$, then we obtain

$$
\begin{aligned}
\Delta=\frac{w^{\prime \prime}(1)}{\left[1+\frac{2 g}{L} w^{\prime}(1)^{2}\right]^{3 / 2}}= & a\left[\int_{0}^{2} \frac{3 s^{2} \mathrm{~d} s}{2\left(1-\Delta s^{2}(s-2)^{2}\right)^{2}} .\right. \\
& \left.-\int_{1}^{2} \frac{6(s-1) \mathrm{d} s}{\left(1-\Delta s^{2}(s-2)^{2}\right)^{2}}\right]
\end{aligned}
$$

For Eq. (34), the variation of the parameter $a$ with the maximum deflection $\Delta$ is computed with a numerical integration (Matlab) in Fig. 5, and the two critical values are obtained as

$$
\Delta_{\mathrm{PI}}=0.38 \text { and } a_{\mathrm{PI}}=0.1726 \text {. }
$$




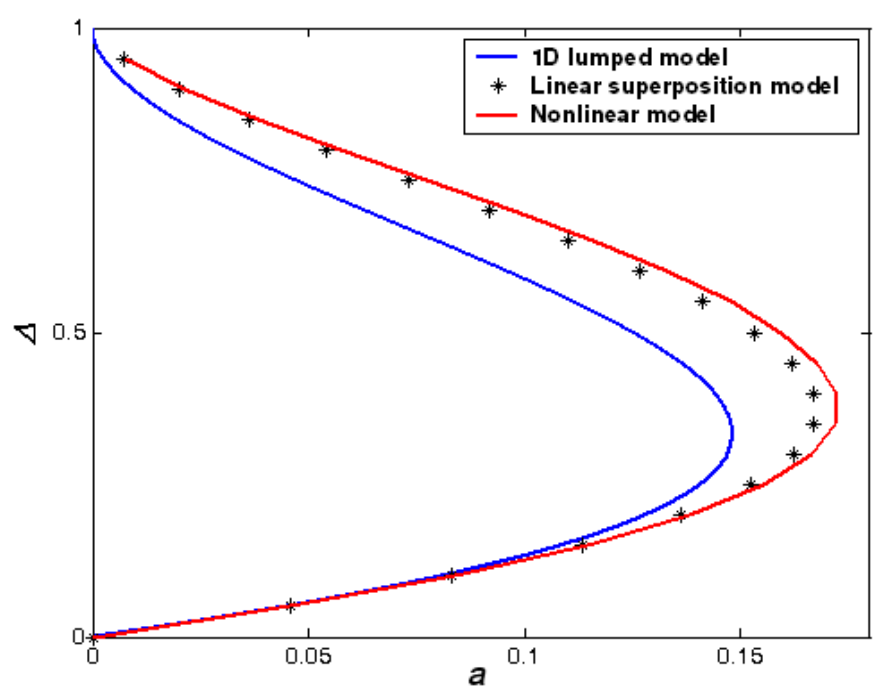

Figure 5. Variation of the maximum deflection $\Delta$ with the parameter of three different models for the fixed-fixed switch.

\section{Comparison and conclusion}

The three different theoretical models, namely 1D lumped model, linear supposition model and the planar model, are discussed and compared to study the pull-in phenomenon about the MEMS (or NEMS) switches. According to the discussion in Sections 2.2 and 2.3 for the cantilever switch, the same result can be obtained from the linear superposition model and the planar model. Then we do not refer to the planar model in Section 3 for the fixed-fixed structure. The results for these models are compared with the nonlinear beam model. For this model, we not only directly use the nonlinear beam model, but also put forward a more accurate assumption for the deflection of the beam by requesting the deflection function to satisfy all the boundary conditions.

Figures 4 and 5 give the variation of the maximum deflection $\Delta$ with the parameter $a$ about three different models, which are 1D lumped model, linear superposition model and nonlinear model. Figures 4 and 5 are for cantilever and fixed-fixed switches, respectively. From these two figures, we notice that both stable and unstable equilibrium points exist for a given dimensionless parameter $a$ which is related to the applied voltage $V$. Equilibrium state does not exist when $a>a_{\mathrm{PI}}$. The critical pull-in value $a_{\mathrm{PI}}$ is different for different models or different structures. The pull-in parameters $a_{\mathrm{PI}}$ and $\Delta_{\mathrm{PI}}$ are listed in Table 1. It is known from this table that the error of the pull-in parameters is large between 1D lumped model and the nonlinear beam model. The large error for 1D lumped model is because the denominator of the electrostatic force is diminished when the electrostatic force is computed as a concentrated force at the maximum deflection along the beam. The linear superposition model and the slender planar model not only have the same result but also exist very little error of the pull-in values with the nonlinear beam model because these two models all consider the variation of electrostatic force with the beam's deflection. The error brought by these two models is attributed to that the boundary conditions are not completely satisfied when computing the numerical integration for the deflection.

When these models are used to discuss the pull-in phenomenon which is a strong nonlinear problem [32-34], special attention should be paid to the effect of error on the practical design. 
In order to get the more accurate parameter for design, it is suggested to use the linear superposition model. Nevertheless, the planar model should be used if the dynamical behavior of MEMS switches is discussed.

Cantilever switch $\quad$ Fixed-fixed switch

Different models

1D lumped model

Linear superposition model

Nonlinear model

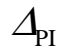

0.33

0.47

0.47

$$
a_{\mathrm{PI}}
$$

0.1481

0.2284

0.2267

$$
\Delta_{\mathrm{PI}}
$$$$
a_{\mathrm{PI}}
$$

0.33

0.1481

0.38

0.1675

0.38

0.1726

Table 1 . The pull-in values $\Delta_{\mathrm{PI}}$ and $a_{\mathrm{PI}}$ for three different models and two different structures.

\section{Acknowledgments}

WHL was supported by the National Natural Science Foundation of China (Grant No. 10602062) and YPZ was jointly supported by the National Basic Research Program of China (973 Program, Grant No. 2007CB310500) and the National High-tech R\&D Program of China (863 Program, Grant No. 2007AA04Z348).

\section{References}

[1] Osterberg PM. Electrostatically actuated microelectromechanical test structures for material property measurements, $\mathrm{Ph}$. D. dissertation, MIT, Cambridge, MA (1995).

[2] Osterberg PM and Senturia SD. M-TEST: A test chip for MEMS material property measurement using electrostatically actuated test structures. J. Microelectromech. Syst., 6: 257-265 (1997).

[3] Pamidighantam S, Puers R, Baert K and Tilmans HAC. Pull-in voltage analysis of electrostatically actuated beam structures with fixed-fixed and fixed-free end conditions. J. Micromech. Microeng., 12: 458-464 (2002).

[4] Johnstone RW and Parameswaran M. Theoretical limits on the freestanding length of cantilevers produced by surface micromachining technology. J. Micromech. Microeng., 12: 855-861 (2002).

[5] Ramezani A, Alasty A and Akbari J. Influence of van der Waals force on the pull-in parameters of cantilever type nanoscale electrostatic actuators. Microsyst. Technol., 12: 1153-1161 (2006).

[6] Yang FQ. Electromechanical instability of microscale structure. J. Appl. Phys., 92: 2789-2794 (2002).

[7] Serry FM, Walliser D and Maclay GJ. The role of the Casimir effect in the static deflection and stiction of membrane strips in microelectromechanical systems (MEMS). $J$. Appl. Phys., 84: 2501-2506 (1998).

[8] Abdel-Rahman EM, Younis MI and Nayfeh AH. Characterization of the mechanical behavior of an electrically actuated microbeam. J. Micromech. Microeng., 12: 759-766 (2002).

[9] Zhang LX and Zhao YP. Electromechanical model of RF MEMS switches. Microsyst. Technol., 9: 420-426 (2003).

[10] Bochobza-Degani O and Nemirovsky Y. Modeling the pull-in parameters of electrostatic actuators with a novel lumped two degrees of freedom pull-in model. Sensors Actuat. A, 97: 569-578 (2002).

[11] Dequesnes M, Rotkin SV and Aluru NR. Calculation of pull-in voltages for carbon-nanotube-based nanoelectromechanical switches. Nanotechnology, 13: 120-131 (2002).

[12] Lin WH and Zhao YP. Casimir effect on the pull-in parameters of nanometer switches. Microsyst. Technol., 11: 80-85 (2005).

[13] Lin WH and Zhao YP. Nonlinear behavior for nanoscales electrostatic actuators with Casimir force. Chaos, Solitons \& Fractals, 
23: 1777-1785 (2005).

[14] Guo JG and Zhao YP. Influence of van der Waals and Casimire forces on electrostatic torsional actuators. J. Microelectromech. Syst., 13: 1027-1035 (2004).

[15] Lin WH and Zhao YP. Dynamic behaviour of nanoscale electrostatic actuators. Chin. Phys. Lett., 20: 2070-2073 (2003).

[16] Guo JG and Zhao YP. Dynamic stability of electrostatic torsional actuators with van der Waals effect. Int. J. Solids Struct., 43: 675-685 (2006).

[17] Roark RJ and Young WC. Formulas for Stress and Strain, New York: McGraw-Hill (1989).

[18] Palasantzas G. Pull-in voltage of microswitch rough plates in presence of electromagnetic and acoustic Casimir forces. J. Appl. Phys., 101: 063548 (2007).

[19] Palasantzas G. Contact angle influence on the pull-in voltage of electrostatic switches in presence of capillary condensation and quantum vacuum effects. J. Appl. Phys., 101: 053512 (2007).

[20] Palasantzas G and De Hosson JTM. Surface roughness influence on the pull-in voltage of microswitches in presence of thermal and quantum vacuum fluctuations. Surf. Sci., 600: 1450-1455 (2006).

[21] Ramezani A, Alasty A and Akbari J. Analytical investigation and numerical verification of Casimir effect on electrostatic nano-cantilevers. Microsyst. Technol., 14: 145-157 (2008).

[22] Esquivel-Sirvent R, Reyes L and Bárcenas J. Stability and the proximity theorem in Casimir actuated nano devices. New J. Phys., 8: 241 (2006).

[23] Dai W and Zhao YP. The nonlinear phenomena of thin polydimethylsiloxane (PDMS) films in electrowetting. Int. J. Nonlinear Sci. Numer. Simul., 8: 519-526 (2007).

[24] Zhang LX, Zhang JW, Zhao YP, et al. Failure modes of doubly supported capacitive RF MEMS switches. Int. J. Nonlinear Sci. Numer. Simul., 3: 353-356 (2002).
[25] Batra RC, Porfiri $M$ and Spinello D. Review of modeling electrostatically actuated microelectromechanical systems. Smart Mater. Struct., 16: R23-R31 (2007).

[26] Sadeghian H, Rezazadeh G and Osterberg PM. Application of the generalized differential quadrature method to the study of pull-in phenomena of MEMS switches. $J$. Microelectromech. Syst., 16: 1334-1340 (2007).

[27] Seydel R. Practical Bifurcation and Stability Analysis: from Equilibrium to Chaos, 2nd ed., Springer-Verlag (1994).

[28] Shou DH and He JH. Application of parameter-expanding method to strongly nonlinear oscillators. Int. J. Nonlinear Sci. Numer. Simul., 8: 121-124 (2007).

[29] Wang SQ and He JH. Nonlinear oscillator with discontinuity by parameter-expansion method. Chaos, Solitons and Fractals, 35: 688-691 (2008).

[30] He JH. The variational iteration method: Reliable, efficient, and promising. Computers and Mathematics with Application, 54: 879-880 (2007).

[31] $\mathrm{He} \mathrm{JH}$ and $\mathrm{Wu} \mathrm{XH}$. Variational iteration method: New development and applications. Computers and Mathematics with Application, 54: 881-894 (2007).

[32] Lin WH and Zhao YP. Influence of damping on the dynamical behavior of the electrostatic parallel-plate and torsional actuators with intermolecular forces. Sensors, 7: 3012-3026 (2007).

[33] Zhang Y and Zhao YP. Nonlinear dynamics of atomic force microscopy with intermittent contact. Chaos, Solitons and Fractals, 34: 1021-1024 (2007).

[34] Lin WH and Zhao YP. Stability and bifurcation behavior of electrostatic torsional NEMS varactor influenced by dispersion forces. J. Phys. D: Appl. Phys., 40: 1649-1654 (2007). 